\title{
Anionic linear-globular dendrimer-cis-platinum (II) conjugates promote cytotoxicity in vitro against different cancer cell lines
}

This article was published in the following Dove Press journal:

International Journal of Nanomedicine

24 December 2009

Number of times this article has been viewed

\author{
Ismaeil Haririan ${ }^{1,6}$ \\ Mohammad Shafiee \\ Alavidjeh' \\ Mohammad Reza \\ Khorramizadeh ${ }^{2}$ \\ Mehdi Shafiee Ardestani ${ }^{3}$ \\ Zohre Zarei Ghane ${ }^{4}$ \\ Hassan Namazi ${ }^{5}$ \\ 'Department of Pharmaceutics, \\ Faculty of Pharmacy, ${ }^{2}$ Department \\ of Medical Biotechnology, School of \\ Advanced Medical Biotechnology, \\ ${ }^{3}$ Department of Medicinal Chemistry, \\ Faculty of Pharmacy, ${ }^{4}$ Department of \\ Pathobiology, School of Public Health, \\ Tehran University of Medical Sciences, \\ Tehran, Iran; ${ }^{5}$ Faculty of Chemistry, \\ Laboratory of Natural Carbohydrates \\ and Biopolymer, University of Tabriz, \\ Tabriz, Iran; ${ }^{6}$ Biomaterials Research \\ Center (BRC), Tehran, Iran
}

\begin{abstract}
Due to their unique properties, Anticancer dendrimer-based drugs have been displaying promising results in both in vitro and in vivo in the treatment of cancerous cells, as compared to the traditional polymers. In this report, two conjugates $(\mathrm{G} 1+\mathrm{Pt}$ and $\mathrm{G} 2+\mathrm{Pt})$ of cisplatin [cis-diaminedichloroplatinum; (CDDP)] with two generations (G1, G2) of a biocompatible anionic dendrimer were prepared in an aqueous media. Their potential cytotoxic effects, in two sensitive cancer cell lines HT1080 and CT26 together with one resistant cancer cell line SKOV3, using MTT (methyl thiazolyl tetrazolium) assay were examined. Hemolytic impacts and cell death mechanisms of the conjugates on human blood and HT1080 cell line were also investigated. The conjugate G2+Pt showed greater toxicity up to $9 \times$ and $2 \times$ in the sensitive and resistant cell lines $\left(\mathrm{IC}_{50}\right.$ comparison, inhibitory concentration) respectively when compared to the parent drug. The G1+Pt conjugate showed greater toxicity only in the sensitive HT1080 (2×) and CT26 (3.7×) cell lines. Moreover, the G1+Pt conjugate was less toxic approximately one third of the cisplatin in SKOV3 after $48 \mathrm{hrs}$ of incubation. In summary, the G2+Pt conjugate had greater toxicity than the G1+Pt conjugate and cisplatin, based on the in vitro results. Approximately the same hemolysis behavior was observed for both conjugates and cisplatin. Both apoptosis and necrosis mechanisms (about $2 \times$ more than cisplatin) were attributed to conjugates and cisplatin in a direct correlation between the concentration and the degree of cell death. In conclusion, these conjugates with such high potency and minimum hemolysis would be suitable candidates for use against these cancerous cell lines as efficient and novel antitumor agents.
\end{abstract}

Keywords: cis-platinum (II), dendrimer, in vitro cytotoxicity, hemolysis, apoptosis-necrosis

\section{Introduction}

Macromolecular prodrugs, especially polymer-drug conjugates, which were first proposed by Ringsdorf in the mid 1970s, are the foundation for new types of anticancer entities. ${ }^{1}$ Soon after this, very promising antitumor activities (eg, due to the alteration of parent drug pharmacokinetics/pharmacodynamics) were demonstrated for these structures in preclinical and clinical study phases. ${ }^{2}$ Conventionally, hydrophilic, linear and branched bio- or nonbiodegradable polymers such as natural polymers; the linear polymers of amidoamines, or N-(2-Hydroxypropyl) methacrylamide (HPMA)-copolymers have been utilized as carriers for anticancer agents. However, these polymers have shown some drawbacks, eg, unpredictable and uncontrollable pharmacokinetic/pharmacodynamic behaviors due to their inherently high polydispersity index which are due to the heterogeneous products formed. ${ }^{3}$ Today, polymeric structures with very low polydispersity, eg dendrimers, are being preferred as carriers
Correspondence: Ismaeil Haririan Department of Pharmaceutics, Faculty of Pharmacy, Tehran University of Medical Sciences, Tehran, |4|55-645I, Iran

Tel +98 2166482607

Fax +98 2I 66482607

Email haririan@tums.ac.ir 
of antitumor drugs. Dendrimers are a series of polymeric architectures with different chemical and surface-related properties. In comparison to traditional polymers, dendrimers have been claimed to be superior due to their nanoscale (nanometersized polymers) architecture, narrow polydispersity index, and the large number of surface reactive/functional sites with the potentiality for bond formation with therapeutics. ${ }^{4}$

Yet, not all of the dendrimers are suitable in this respect; biocompatible properties like aqueous solubility, biodegradation, low cytotoxicity and nonimmunogenicity properties must be verified for them both in vitro and in vivo. ${ }^{5}$ One variant of these macromolecular prodrugs that has been partially investigated is the dendrimer cisplatin conjugates. ${ }^{6}$ Encouraging outcomes in the treatment of various kinds of cancer cells have been reported for these compounds. ${ }^{7}$ However, one major problem associated with these drug conjugates is the toxicity of the dendrimers themselves. This toxicity is dependant upon the physico-chemical properties of the dendrimers. ${ }^{5}$ Research and development of dendrimer-based antineoplastic prodrugs can be accelerated with the use of biologically safe dendrimers, as carriers for conventional anticancer drugs. This is especially pertinent for cancers with drug resistance eg, as a consequence of the reduction in drug uptake. Cisplatin, an anticancer drug (cis-diamminedichloroplatinum with a molecular weight of $300.04 \mathrm{Da}$ ) used extensively in the treatment of many cancers such as ovarian, head, neck, testicular, and small cell lung carcinoma was chosen as a model drug to study its physicochemical interactions with the dendrimers. ${ }^{8}$ The disadvantages of low aqueous solubility, high protein binding that reduce drug potencies, together with the nonspecific or systemic toxicity (eg, nephrotoxicity) and both inherent/or acquired resisitences in many types of treatments have been reported for platinum-derived drugs. ${ }^{9,10}$ Thus, our objective for studying these dendrimer-Pt conjugates was to increase the anticancer potency of cisplatin by increasing its water solubility, decreasing its protein binding, and also reduction its nephrotoxicity with a view to their clinical use. It is postulated that these advantages could be gained by displacing the transient, easily alterable chemical agents of cisplatin (ie, chloride ions) with groups such as carboxylate ions on the dendrimer surfaces, so as to form more stable bonds (due to greater thermodynamic stability, steric effects, and ring stability of carboxylate ions versus the chloride ions) with cisplatin and prevent nonspecific and undesirable reactions with strong nucleophilic groups (eg, thiols present in plasma proteins or membrane walls of kidney cells) resulting from cisplatin's poor water-stability prior to reaching their sites of actions. ${ }^{11-14}$ Increasing the stability of these bonds would preclude undesirable protein binding and systemic toxicities that are associated with cisplatin therapy.

Using citric acid in the synthesis of these dendrimers, it was hoped that the conjugates thus formed would be able to overcome the reduced uptake of cisplatin in cell lines with intrinsic and/or acquired resistances. It was hypothesized that this could be achieved by sequestering cisplatin (ie, masking via the linkage process) and supplying the cell's energy needs with the citric acid (a key component in the synthesis of adenosine triphosphate), utilized in the synthesis of the dendrimers. It was hoped that, dendrimers synthesized in this way can carry the drug into the cells without any intervention of the resistance mechanisms. So, two generations of anionic, water-soluble, linear-globular dendrimers G1 (MW 1000 Da) and G2 (MW 2000 Da), where poly ethylene glycol (PEG) is the core and citric acid is the periphery, were selected for the subsequent couplings with cisplatin.

These dendrimers had been synthesized before by Namazi and colleagues. ${ }^{15,16}$ and in our previous studies any in vitro cytotoxicity, immunogenicity, and hemotoxicity were not observed up to the concentration of $0.5 \mathrm{mg} / \mathrm{mL}$ (Alavidjeh and colleagues.unpublished data). First, In this study, surface conjugations and surface properties of the dendrimers (eg, size and zeta potential distribution) as a result of the reaction with cisplatin were examined using Fourier transform infrared (FT-IR) spectroscopy and dynamic light scattering (DLS ) technique. The reaction kinetics of cisplatin in the absence and presence of the dendrimers was investigated by measuring the chloride ions released using an ion selective electrode. The amount of drug loading on each of the conjugates was determined by atomic absorption spectroscopy (AAS). The in vitro release of cisplatin was examined in a two buffer media with two pHs of 7.4 and 5.4, simulating the conditions of plasma and secondary lysosomes, for 72 hours to estimate the stability and strength of the conjugates under those conditions. The in vitro cytotoxic potential of the conjugates was examined using sensitive cell lines HT1080, CT26 and a partially resistant cell line SKOV3. The hemolytic behavior of each of the conjugates was examined at different concentrations and a variety of durations to to assess the hemotoxicity of these compounds versus free cisplatin. Finally the mechanisms of cell death induced by the conjugates and free cisplatin, in two different concentrations, were studied by flow cytometry method as these mechanisms are considered important in the final outcome of cancer therapy. ${ }^{17}$ In summary, the results of these studies would be able to assist in the primary screening of more potent macromolecular prodrugs with less systemic side effects. 


\section{Materials and methods Materials}

The dendrimers (G1, [1000 Da] and G2, [2000 Da]) had previously been prepared by the method of Namazi and colleagues. ${ }^{15,16}$ Cisplatin (II) (MW 300.4 Da) and methyl thiazolyl tetrazolium (MTT) powder were purchased from SigmaAldrich, (Sigma-Aldrich Inc, St. Louis, MO, USA). Dialysis bag (molecular weight cut-off, 100-500 Da) was purchased from Spectrumlabs (Spectra/por ${ }^{\circledR}$, Rancho Dominguez, CA, USA). Cell lines HT1080 (Human fibrosarcoma, ATCC, CCL-121), SKOV3 (Human ovarian, NCBI code; C209), CT26 (fibroblast, NCBI code; C532) were purchased from The Pasteur Institiute (Tehran, Iran), and AnnexinV-PI kit was obtained from Roche (Roche company, AnnexinV-FLUOS ${ }^{\circledR}$, Basel, Switzerland).

\section{Preparation of the cisplatin derivatives}

The dendrimers with carboxylate end groups were reacted with cisplatin in two different molar ratios ie, 1:2 and 1:3. Typically, $180 \mathrm{mg}$ of cisplatin $\left(6 \times 10^{-4} \mathrm{~mole}\right)$ and the dendrimers, G1 (2-3 $\times 10^{-4}$ mole), G2 (2-3 $3 \times 10^{-4}$ mole $)$, were dissolved in $120 \mathrm{~mL}$ and $60 \mathrm{~mL}$ of double deionized water (D.D.W) separately for each of the reactions. Each dendrimer solution was then partially neutralized using $0.01 \mathrm{M} \mathrm{NaOH}$ solution, to provide the nucleophilic carboxylate end groups, and then freeze-dried for subsequent reactions with cisplatin. The dendrimer solutions, in different molar ratios, were added dropwise to the cisplatin solution with constant mild stirring. The stirring was continued, in the dark, for 18 hours. At the end of the reaction the mixtures were transferred to a dialysis bag and dialyzed against D.D.W for 72 hours to separate the unreacted cisplatin from the formed conjugates. A sample from the filtrate was transferred to a TLC plate and tested for the absence of cisplatin as mentioned in the USPXXIV Pharmacopea (developer: acetone and $1 \mathrm{M}$ nitric acid (90:10); spray reagents: stannous chloride, hydrogen chloride, and potassium iodide). When no platinum release was observed after $72 \mathrm{hrs}$, the samples inside the dialysis bag were lyophilized and kept at $-20{ }^{\circ} \mathrm{C}$ for further analyses. The schematic structural diagrams of the dendrimers, cisplatin, and the possible formed conjugates were depicted in Figure 1.

\section{Chloride release}

In order to have a comparison between the kinetics of the reaction of cisplatin with or without the dendrimers in the reaction media, the rate of chloride release was measured from cisplatin in both the absence and presence of the dendrimers in D.D.W using an ion selective electrode (Metrohm ${ }^{\circledR}$, Oberdorfstrasse, Switzerland).

\section{Drug loading}

The drug loading range was determined by atomic absorption spectroscopy (AAS) method (SpectrAA240, Varian Inc, Palo Alto, CA, USA) for each of the derivatives in two different molar ratios.

\section{Platinum release}

The dendrimer-Pt conjugates $(10 \mathrm{mg} / \mathrm{mL})$ were each dissolved in two different Sorenson's buffers $\mathrm{pH} 7.4$ and 5.4, to simulate the physiological media. The resultant samples were then dialyzed against the same buffers, at $37^{\circ} \mathrm{C}$ for $72 \mathrm{hrs}$. After each time interval a sample was taken to determine the platinum content of the conjugates by the AAS method.

\section{Size and zeta potential distribution}

Each of the dendrimers and conjugates $(0.5 \mathrm{mg} / \mathrm{mL})$ was checked for any changes in the size and zeta potential distribution before and after the reaction with the cisplatin by DLS technique (Malvern, Zetasizer Nano ZS, Worcestershire, UK) in D.D.W.

\section{FT-IR studies}

The spectral changes of the dendrimers were examined by Fourier transform infrared (FTIR) spectroscopy (ThermoNicolet, NEXUS 870, Waltham, MA, USA) before and after the conjugation. Through changes in the wavenumbers of the functional groups in the IR spectra, we were able to show the surface conjugation and clarify which groups were involved in the conjugation process.

\section{In vitro toxicity assay}

MTT assay is one the current techniques which is widely used for cell viability measurement. This assay is based on the reduction of MTT to formazan by viable cells. ${ }^{18,19}$ At the end of the incubation times $(24,48 \mathrm{hrs})$ with various concentrations of the conjugates $(0.787,1.575,3.15,6.3 \mu \mathrm{g} / \mathrm{mL}$; equivalent to free cisplatin) and pristine cisplatin, the supernatants of the cells (HT1080, SKOV3, CT26) were removed. MTT solution was added to each well of the plates to give the final concentration of $0.5 \mathrm{mg} / \mathrm{mL}$, and the cells were incubated for an additional 4 hours. Thereafter, the solutions were removed, and the dye was dissolved in $100 \mu \mathrm{l}$ dimethyl sulfoxide; the plates were kept in a dark place for another one hour in order to be ready for spectrophotometric determination. The amount of absorption in each well; that is, 


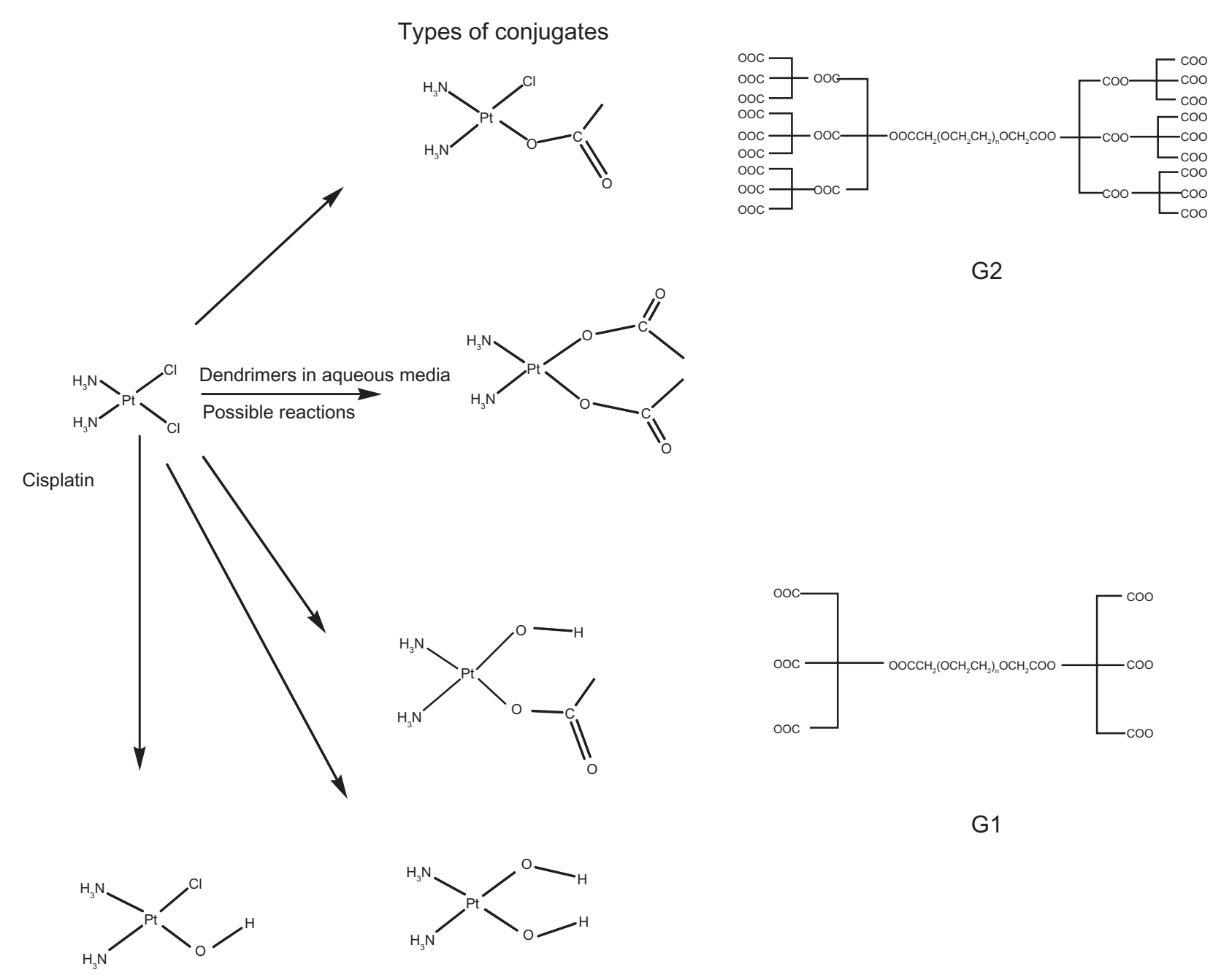

Figure I Schematic diagrams of cisplatin (CDDP), the dendrimers GI, G2 and their possible reactions (generation of different conjugates) in aqueous media.

the conversion of MTT to formazan by metabolically viable cells, was calculated by an automated microplate reader at $570 \mathrm{~nm}$. The results were devided to the absorption of the untreated control culture and expressed as percentage of viable cells.

\section{Hemolysis assay}

Blood samples were taken from un-medicated volunteers (anti-coagulated samples). The final hematocrit fraction was obtained after centrifuging the whole blood at $1000 \mathrm{rpm}$ for $5 \mathrm{~min}$, and discarding the supernatant. The cells were then rinsed three times with cold phosphate buffered saline (PBS 5\% v/v). The conjugates and free cisplatin were then dissolved in PBS and incubated and shaken at $37{ }^{\circ} \mathrm{C}$ and $50 \mathrm{rpm}$ respectively for two hours with the blood sample mixtures, composed of $1: 5 \mathrm{v} / \mathrm{v}$ conjugate solutions to blood to make the final, specified concentrations of the conjugates and cisplatin $(1.575,3.15,6.3 \mu \mathrm{g} / \mathrm{mL}$ weight equivalent to free cisplatin). Following this the mixture was centrifuged at $1000 \mathrm{rpm}$ for $5 \mathrm{~min}$ and the supernatants removed and analysed using a spectrophotometer at $413 \mathrm{~nm}$. The results were then compared to the positive control $(0.1 \% \mathrm{v} / \mathrm{v}$ Triton$\mathrm{X} 100$ in PBS) which produces $100 \%$ hemolysis and negative control which contained only a mixture of blood and PBS as an indicator for the minimum hemoglobin release. ${ }^{19,20}$

\section{Apoptosis-necrosis assay}

Apoptotic and necrotic cells were identified by an AnnexinV-PI Staining Kit. Apoptosis and necrosis have been considered as the two different mechanisms of the cell death. By this technique, we are able to measure and differentiate between these two mechanisms. ${ }^{19,21}$ Following the incubation for 24 hours with two different concentrations of the G1+Pt, G2+Pt and free cisplatin ( 0.75 and $1.5 \mu \mathrm{g} / \mathrm{mL}$, the weight equivalent to cisplatin) with the HT1080 Cells, and they were washed three times with PBS, trypsinized, and transferred to a new tube. 
Following this the cells were centrifuged at $200 \times \mathrm{g}$ for 5 minutes, stained using trypan blue and then counted using a hemocytometer, Approximately $10^{6}$ cells were analyzed for each flow cytometry run. The cells, both treated and untreated ones, were then incubated with the AnnexinV-PI (propidium iodide) reagent buffer for 15 minutes at room temperature. Following the incubation the samples were diluted with $500 \mu \mathrm{L}$ of the incubation buffer for flow cytometry analysis. The percentage of the treated apoptotic and necrotic cells versus the untreated control cells was identified by the flow cytometry (Partec, GmbH Munster, Germany) method with Flomax software (version 2.4) at suitable excitation (488 nm) and emission wavelengths $(518 \mathrm{~nm})$ for Annexin-V detection and a band pass filter greater than $600 \mathrm{~nm}$ for propidium iodide (PI) detection according to the suggested protocol in the kit for FITC-PI detection.

\section{Statistical analysis}

All the experiments were conducted in triplicate and the results were compared using one-way ANOVA for mean comparison of more than two groups with SPSSsoftware. ${ }^{11}$ Results were reported as significant for $P<0.05$.

\section{Results}

\section{Chloride release}

The rate of chloride ion release from the cisplatin in the presence of the dendrimers in aqueous media showed a higher $(\times 2)$ and approximately the same as the free cisplatin in the absence of the dendrimers at $18 \mathrm{hrs}$ after the beginning of the reaction (Figure 2). Therefore, it was concluded that the rate of the reaction (chloride release) with cisplatin in the aqueous media containing the dendrimers was higher than in the aqueous media without the dendrimers. This may result from the contribution of the carboxylate ions together with the water molecules in displacing of the chloride ions from cisplatin.

\section{Drug loading}

Almost the same amount of drug loading ( $\sim 6 \%$ weight of platinum/weight of the conjugates) was gained for each of the dendrimers irrespective of their generations and molar ratios (Table 1). This might result from the fact that the effect of the more functional groups in the $\mathrm{G} 2$ can be neutralized by its more associated steric repulsion in comparison with the G1.

\section{In vitro release of platinum}

The amount of platinum release from the two conjugates at differing $\mathrm{pHs}(\mathrm{pH} 7.4$ and $\mathrm{pH} 5.4$ were selected in order to mimic the physiological media existing in the plasma and secondary lysosomes, respectively) showed both a time and concentration dependent behavior. According to the release amounts, the $\mathrm{G} 2+\mathrm{Pt}$ was a little more stable (less than $3 \%$ ) than the $\mathrm{G} 1+\mathrm{Pt}$. On the whole, the release rate in the acidic $\mathrm{pH}(5.4)$ was slightly greater and faster (about $2 \%$ ) than the neutral $\mathrm{pH}$ (7.4) for both conjugates. This phenomenon can be explained due to the catalytic effect of the acidic conditions on the ease of displacement of water molecules with chloride or carboxylate ions inside the cisplatin cavity. This reduction in the release rate of cisplatin in neutral condition ( $\mathrm{pH} \mathrm{7.4)}$ is very important in preserving a stable bond until reaching the target site (tumor) and therefore prevent unwanted and

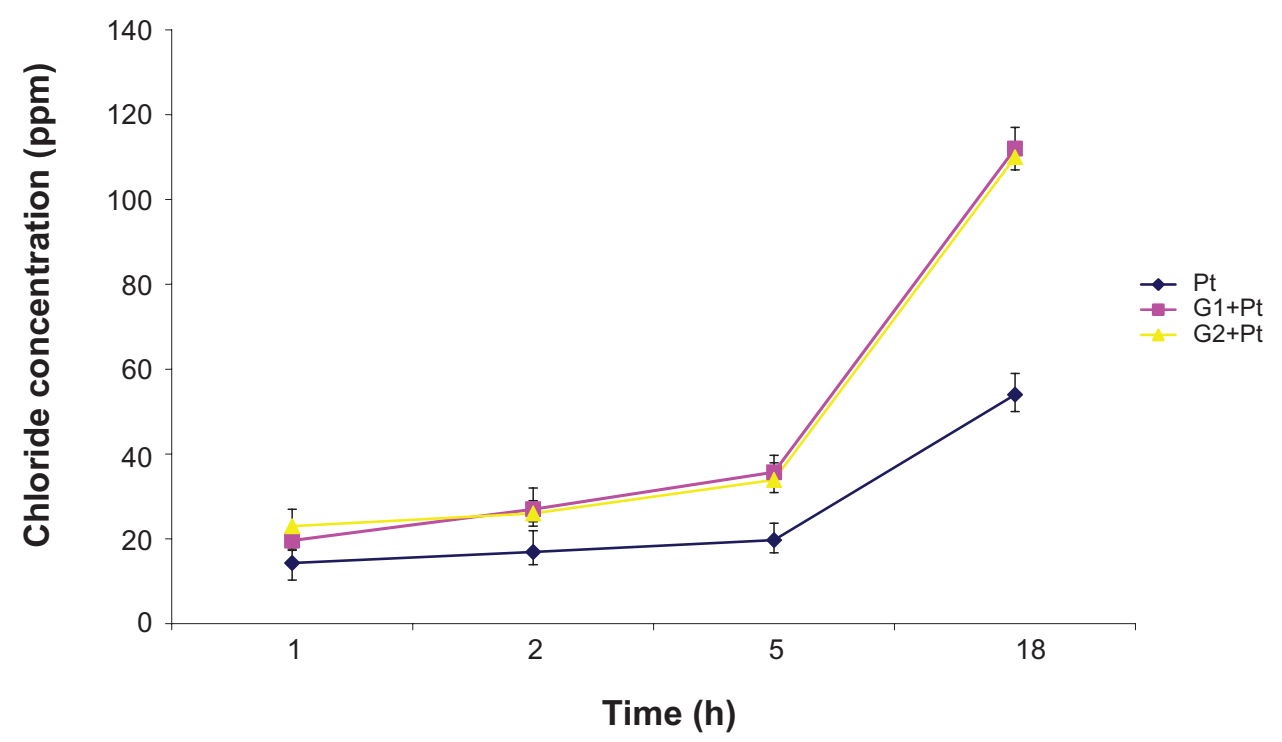

Figure 2 The comparative release of chloride ions from cisplatin (CDDP) alone and in reaction with the dendrimers GI and G2. 
Table I The weight percent of platinum in the conjugates at different ratios of the dendrimers to cisplatin

\begin{tabular}{lll}
\hline Pt derivatives & $\begin{array}{l}\text { Dedrimers: } \\
\text { Pt (CDDP) Ratios }\end{array}$ & $\begin{array}{l}\text { Pt content } \\
\text { in wt \% (mean } \pm \text { SE) } \\
\text { AAS method (flame) }\end{array}$ \\
\hline $\mathrm{GI}+\mathrm{Pt}$ & $\mathrm{I}: 2$ & $5 \pm 0.2 \mathrm{I}$ \\
$\mathrm{GI}+\mathrm{Pt}$ & $\mathrm{I}: 3$ & $6 \pm 0.33$ \\
$\mathrm{G} 2+\mathrm{Pt}$ & $\mathrm{I}: 2$ & $6 \pm 0.21$ \\
$\mathrm{G} 2+\mathrm{Pt}$ & $\mathrm{I}: 3$ & $7 \pm 0.15$ \\
\hline
\end{tabular}

nonspecific toxicities resulting from out of place cisplatin release in the blood circulation. On the whole, the maximum released amount of platinum (approximately $8 \%$ of the total) was observed in the conjugate $\mathrm{G} 1+\mathrm{Pt}$ at $\mathrm{pH} 5.4$ (Figure 3).

\section{Size and zeta potential distribution}

As can be observed from Table 2, when the conjugates were formed from the dendrimers, an increase in the size/poly dispersity and a decrease (negative charge) in the zeta potential were seen in the conjugates as contrasted with the dendrimers. The difference in the size was more marked $(\sim 3 \times)$ in the $\mathrm{G} 1+\mathrm{Pt}$ conjugate, while the variance in the zeta potential distribution was more drastic $(\sim 3 \times)$ in $\mathrm{G} 2+\mathrm{Pt}$ conjugate. This may result from the more negative groups being available at its surface. These phenomena can result from the crosslinking of the dendrimers with cisplatin which intercalates between two of the dendrimer molecules and also masks the negative charges (due to carboxylate ions) of the dendrimers for the reaction with cisplatin, respectively. ${ }^{22}$

\section{FT-IR studies}

In addition to typical peak characteristics of chemical structures of the dendrimers, FT-IR spectra of the conjugate products showed distinct alterations in the spectra (Figure 4) indicating the conjugation at the dendrimer surfaces via carboxylate groups. For example, the marked increase in the $-31 \mathrm{~cm}^{-1}$ wavenumber of IR spectra of the G1 from $1577.81 \mathrm{~cm}^{-1}$ (assignable to asymmetric stretching of-COO-) to $1608.53 \mathrm{~cm}^{-1}$ for the $\mathrm{G} 1+\mathrm{Pt}$ may have resulted from the reaction of oxygen (as a strong base) of the carboxylate (-COO-) ion with platinum (as a weak Lewis acid). This type of bond polarizes, deshields the carbon atom of the-COO-group, this induces a small positive charge on it, and consequently increases the possibility of the carbonyl group having a greater strength and wavenumber. Such an increment (about $25 \mathrm{~cm}^{-1}$ ) was also observed in the $\mathrm{G} 2$, a shift from $1593.17 \mathrm{~cm}^{-1}$, related to asymmetric stretching of the - COO- group of the G2, to $1618.87 \mathrm{~cm}^{-1}$ for the $\mathrm{G} 2+\mathrm{Pt}$. Moreover, the appearance a doublet bands near $3283 \mathrm{~cm}^{-1}$ and $3247 \mathrm{~cm}^{-1}$ for each of the G1+Pt and G2+Pt conjugates in comparison with the $\mathrm{G} 1$ and $\mathrm{G} 2$ dendrimers that is related to the stretching of the N-H groups of cisplatin.

\section{In vitro cytotoxicity assay}

As can be seen from Figure 5, the G2+Pt conjugate was more toxic than the parent cisplatin in all cell lines, times, and concentration range. The cytotoxic strength of some conjugates like $\mathrm{G} 2+\mathrm{Pt}$ based on $\mathrm{IC}_{50}$ calculation (the concentration which inhibits $50 \%$ growth against the

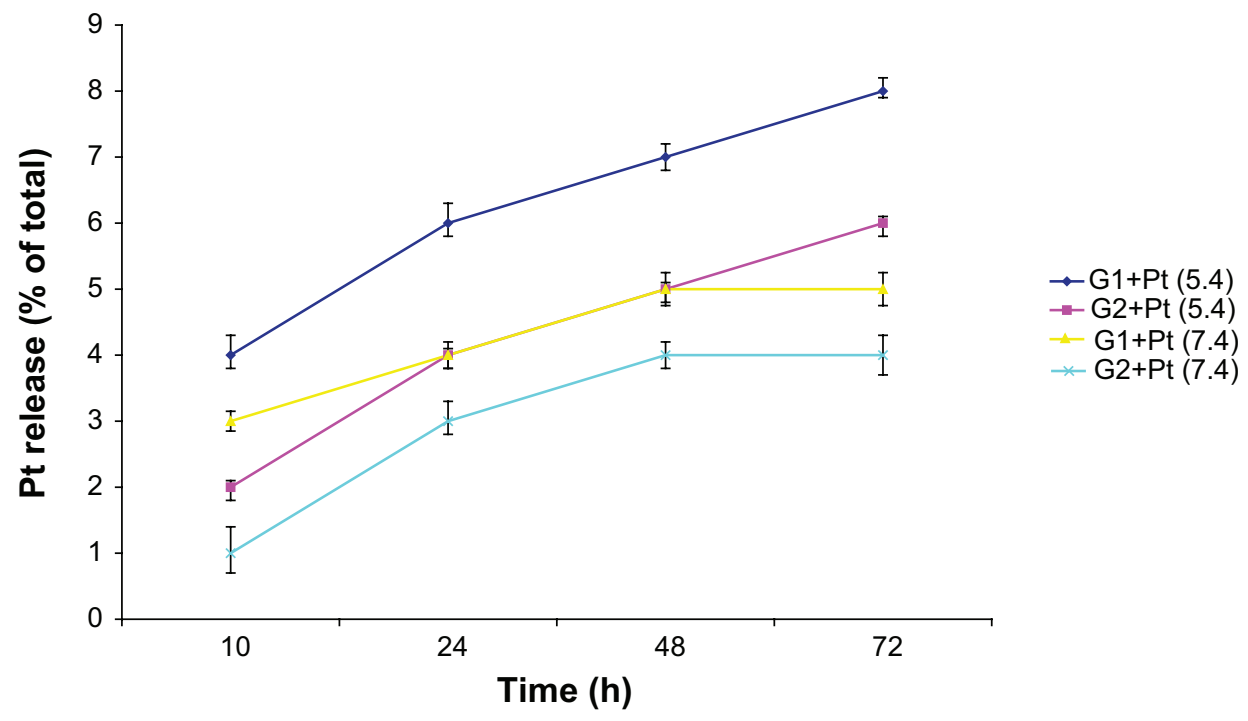

Figure 3 In vitro release of platinum from the GI+Pt and G2+Pt conjugates at two different $\mathrm{pHs}$. Abbreviation: h, hours. 
Table 2 Size and zeta potential distributions of the dendrimers and their related conjugates after reaction with cisplatin (CDDP)

\begin{tabular}{lll}
\hline $\begin{array}{l}\text { Types of the dendrimers } \\
\text { and their derivatives }\end{array}$ & $\begin{array}{l}\text { Size distribution, } \\
\text { mean(nm) } \pm \text { width }\end{array}$ & Zeta potential (mv) \pm width \\
\hline $\mathrm{GI}$ & $225 \pm 32.6(100 \%)$ & $-17.6 \pm 9.68(100 \%)$ \\
$\mathrm{GI}+\mathrm{Pt}$ & $710 \pm 540(94.5 \%) ; 4320 \pm 873(5.5 \%)$ & $-16.4 \pm 5.16(100 \%)$ \\
$\mathrm{G} 2$ & $85.1 \pm \mathrm{II}(100 \%)$ & $-67 \pm 9.70(78.5 \%) ;-4 I \pm 2.62(8.1 \%) ;-7.4 \pm 4.38(8 \%)$ \\
$\mathrm{G} 2+\mathrm{Pt}$ & $\mathrm{I} 1 \mathrm{I} \pm 47.3(100 \%)$ & $-20.9 \pm 4.24(85.2 \%) ;-6.58 \pm 1.42(14.8 \%)$ \\
\hline
\end{tabular}

maximum amount) with nonlinear regression (GraphPad, ${ }^{\circledR}$ version 4) was $9 \times$ greater in HT1080 (24 hrs) and CT26 (48 hrs) cell lines in comparison with free cisplatin while the conjugate $\mathrm{G} 1+\mathrm{Pt}$ showed a higher maximum toxicity of $3.7 \times$ in CT26 cell line in comparison with the cisplatin after $48 \mathrm{hrs}$ of incubation. In addition, the G1+Pt showed less toxicity in SKOV3 cell line increased to one third of free cisplatin after 48 hrs of incubation, while the same potency was observed for SKOV3 and free cisplatin after 24 hrs of incubation. The G1+Pt also showed less toxicity in CT2 6 cell line after $24 \mathrm{hrs}$ of incubation. The $\mathrm{IC}_{50}$ values are displayed in Figure 6.



Figure 4 FT-IR spectra of the dendrimers before and after the reaction with cisplatin (CDDP). IR: (A) GI, (B) GI+Pt, (C) G2, (D) G2+Pt. Abbreviations: FTIR, Fourier transform infrared; IR, infra-red. 


\section{Hemolysis assay}

Based on the results gained from the hemoglobin release from the erythrocytes, only the G2+Pt conjugate at the concentration of $6 \mu \mathrm{g} / \mathrm{mL}$ showed a slightly more hemoglobin release about $1.5 \times$ in comparison with the negative control $(P<0.05)$. The rest of the conjugates did not show any statistical difference $(P>0.05)$ against the negative control over the concentration range. Favourable hemocompatibility was found for these conjugates similar to cisplatin (Figure 7).

\section{Apoptosis-necrosis assay}

Apoptosis-necrosis assay was carried out at two moderately toxic concentrations $(0.75$ and $1.5 \mu \mathrm{g} / \mathrm{mL})$ in order
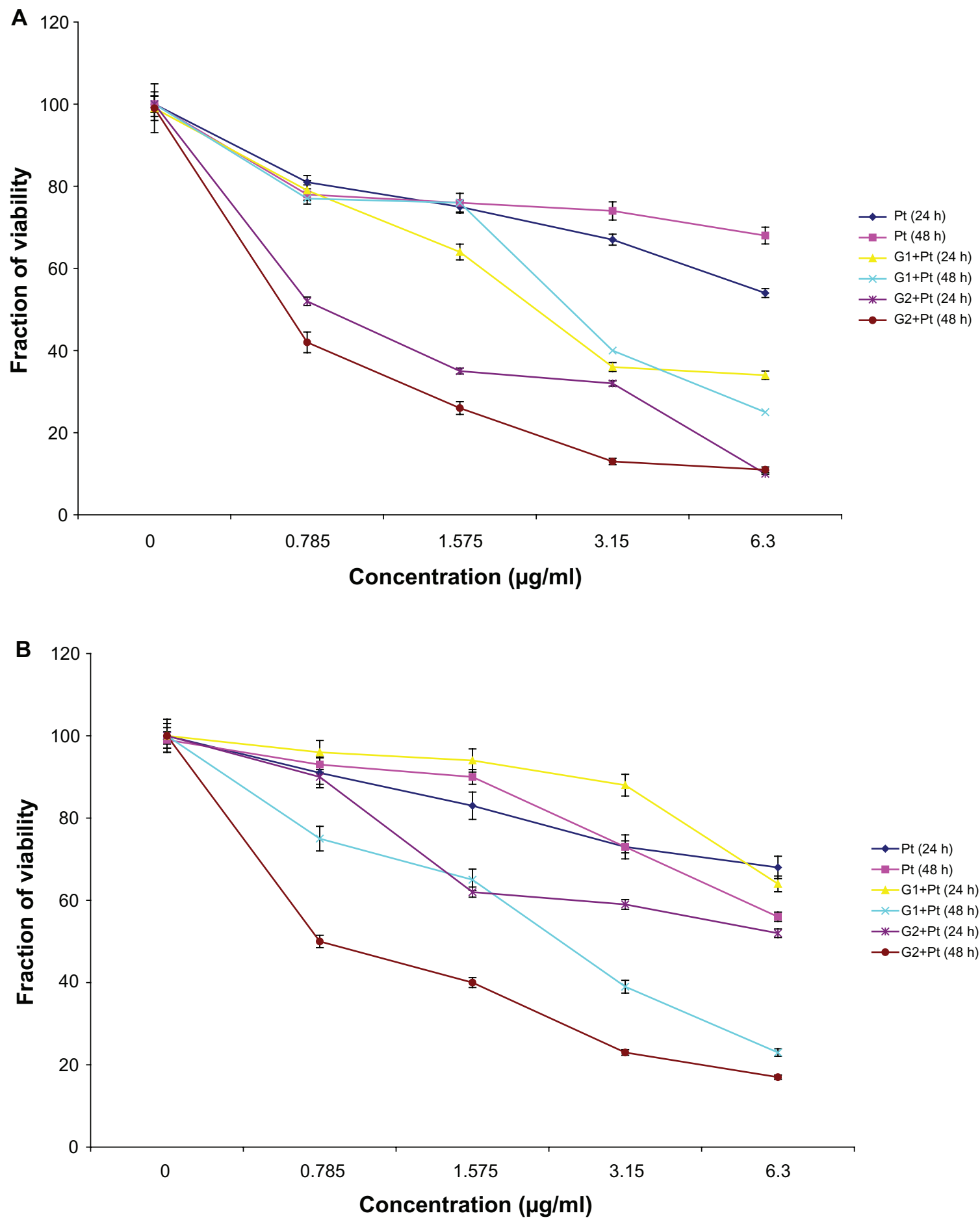


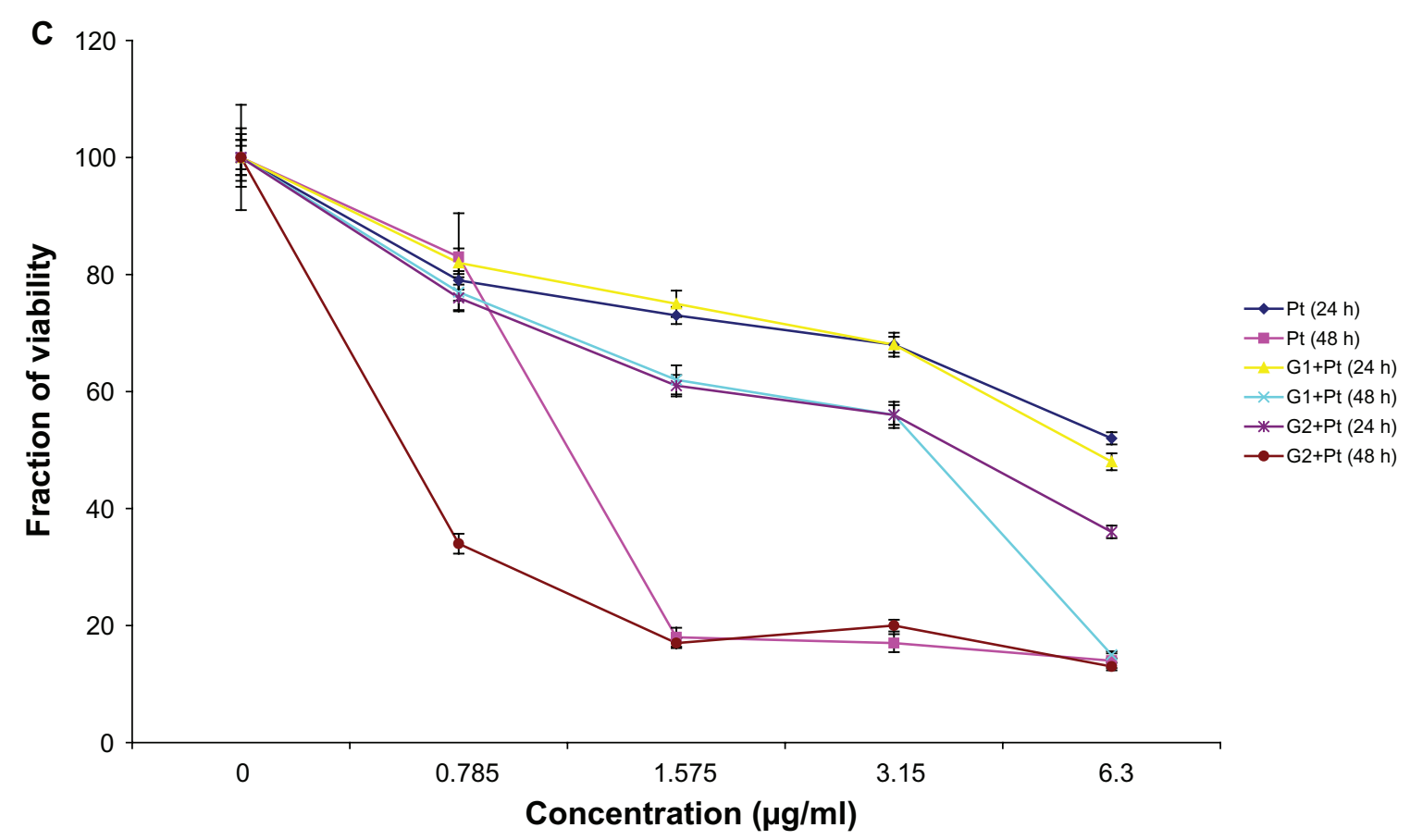

Figure 5 Viability of the different cancer cell lines according to the mitochondrial activity of the cells incubating with different kinds of Pt derivatives and free cisplatin (CDDP) after 24 and 48 hrs. (A) HTI 080 cell line, (B) CT26 cell line, (c) SKOV3.

to determine and differentiate between the two cell death mechanisms induced by the conjugates and free cisplatin. These death mechanisms are considered crucial in determining the final treatment, eradication, and recurrence of the tumor cells. ${ }^{23}$ From the results, a direct correlation between the concentration and cell death was observed for the derivatives and free cisplatin; ie, the higher the concentration the greater the degree of cell death. Greater toxicity was observed for the $\mathrm{G} 2+\mathrm{Pt}$ complex, and increased necrosis was also seen for this conjugate. Generally, the amount of apoptosis and necrosis, was higher nearly $8 \%,(\sim 2 \times)$ for the conjugates than those of cisplatin. The detailed results are presented in the Figure 8.

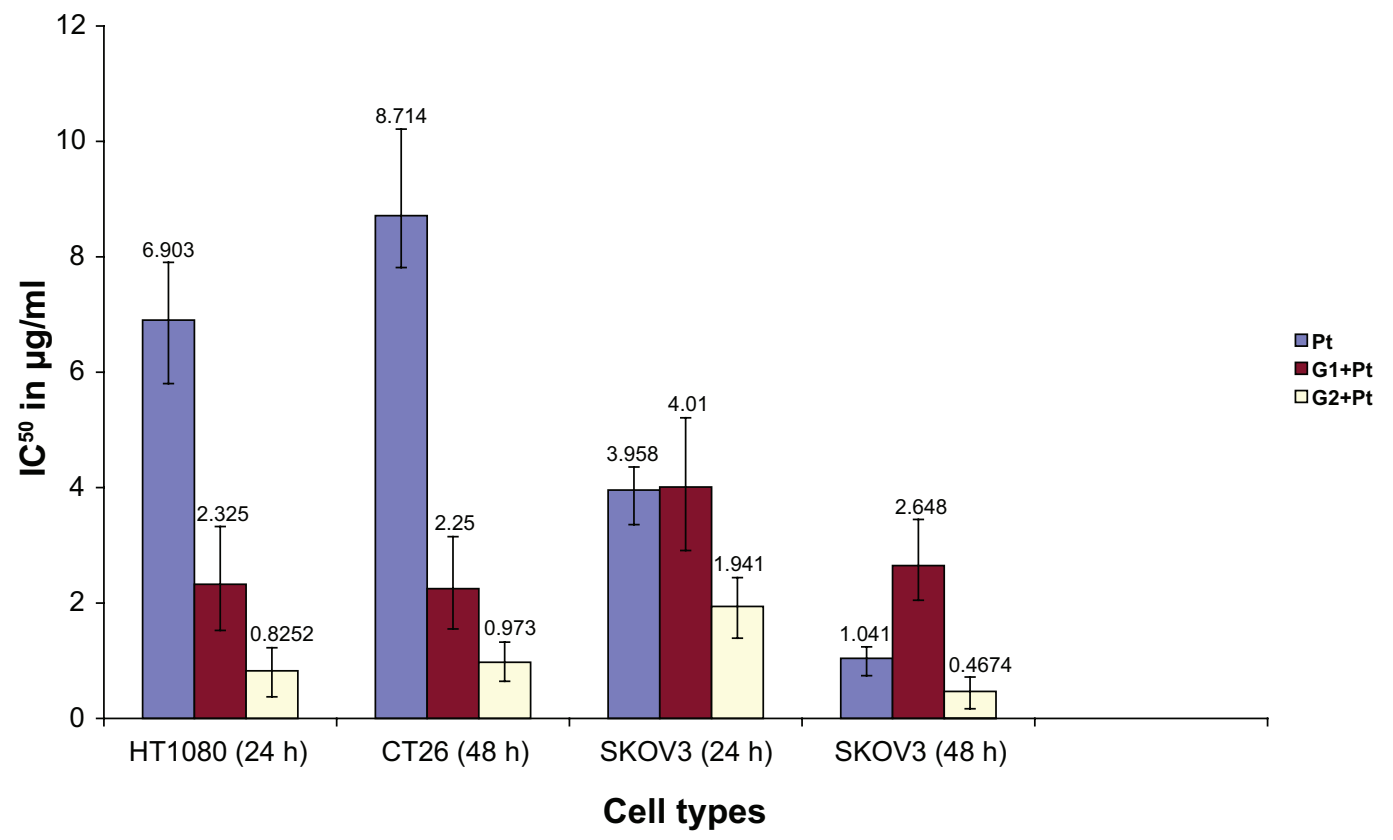

Figure 6 The $I C_{50}$ of some of the conjugates and free cisplatin (CDDP) in different cancer cell lines at different times. Abbreviation: h, hours. 


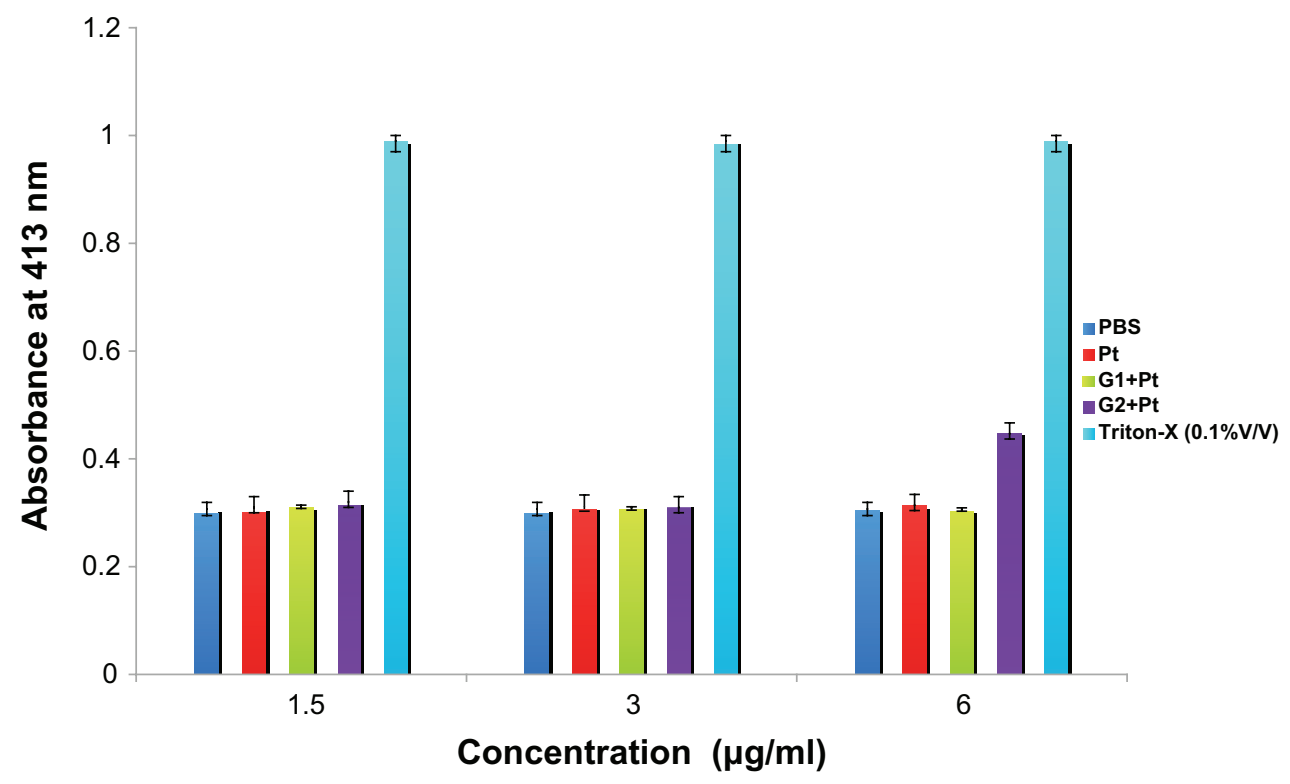

Figure 7 In vitro release of hemoglobin from human erythrocytes in contact with various concentrations of free cisplatin (CDDP) and Pt derivatives after 2 hrs of incubation.

\section{Discussion}

In spite of using many kinds of polymers (traditional or nontraditional types) as drug carriers in the conjugation technique, only a small number of them have shown in vitro toxicity. ${ }^{24}$ The ultimate cytotoxic actions of many types of these conjugates are met when they are used in vivo as an example the polymer-drug conjugates are very stable and not able to release the specified drug when they are inside the tumor cell environment for their final action. ${ }^{25}$ In this study we have shown the synergistic toxic effect of the anionic linear-globular dendrimers (with terminal citric acid groups) conjugated to the surface of cisplatin. This action resulted in the more aqueous solubility of the conjugates as compared to the parent drug. The dendrimers themselves did not show any significant toxicity at the concentrations range used. These observations based on in vitro cytotoxicity $\left(\mathrm{IC}_{50}\right.$ comparison) displayed that the G2+Pt conjugate showed a drastic increase in cytotoxicity in the sensitive cell line HT1080 (up to $8.4 \times$ and greater after $48 \mathrm{hrs)} \mathrm{and} \mathrm{CT26} \mathrm{(up}$ to $9 \times$ after $48 \mathrm{hrs}$ ) at all concentrations and exposure times used. Additionally continued toxicity was observed in these cell lines after $48 \mathrm{hrs}$ of incubation. Such increased toxicity can be explained by a greater uptake of the conjugates as contrasted with the free cisplatin due to the citric acid content of the conjugates together with the greater demands of cancer cells for such energy sources during the time of incubation. The increased liberation of the drug may be attributed to lysosomal enzymes existing inside the cell which gradually breakdown the bond between cisplatin and the dendrimers.
Similar reasons have been proposed for other polymer-drug conjugates thus far. ${ }^{24}$ The $\mathrm{G} 2+\mathrm{Pt}$ showed greater toxicity $\sim 2 \times$ as compared to the free cisplatin in the moderately resistant SKOV 3 cell line. One of the possible resistance mechanisms of this cell line follows from the reduced uptake of cisplatin. ${ }^{26}$ The G1+Pt conjugate showed an approximately a three fold increase in toxicity in the sensitive cell line HT1080. A similar behavior was observed for CT26 cell line only after $48 \mathrm{hrs}$ of incubation (an increment of $3.7 \times$ ) and much less toxicity was seen in this cell line after only $24 \mathrm{hr}$ incubation time. This trend of increase in toxicity over time in the CT26 cell line can be explained by similar reasons to those mentioned above, ie, the gradual uptake and subsequent liberation of the drug due to action of enzymes inside the cell.

The G1+Pt displayed no toxicity in inherently resistant SKOV 3 cell line as compared to free cisplatin. There was even a reduction ( $1 / 3$ of the parent cisplatin) in toxicity observed for the $\mathrm{G} 1+\mathrm{Pt}$ conjugate after $48 \mathrm{hrs}$ when compared to the $24 \mathrm{hrs}$ incubation. A similar potency profile was observed for the G1+Pt conjugate to cisplatin at $24 \mathrm{hrs}$ of incubation. This may suggest that hitherto unknown compensatory mechanisms could neutralize the synergistic effect of the conjugate during the time.

In general, the $\mathrm{G} 2+\mathrm{Pt}$ conjugate was more toxic than the G1+Pt conjugate. This might possibly be related to the higher citric acid content in the G2+Pt conjugate and consequently easier for the tumoric cells to access this for the supply of energy which is a prerequisite for growth. ${ }^{27}$ The smaller and narrower the size distribution (ie, less aggregation behavior) of 
A
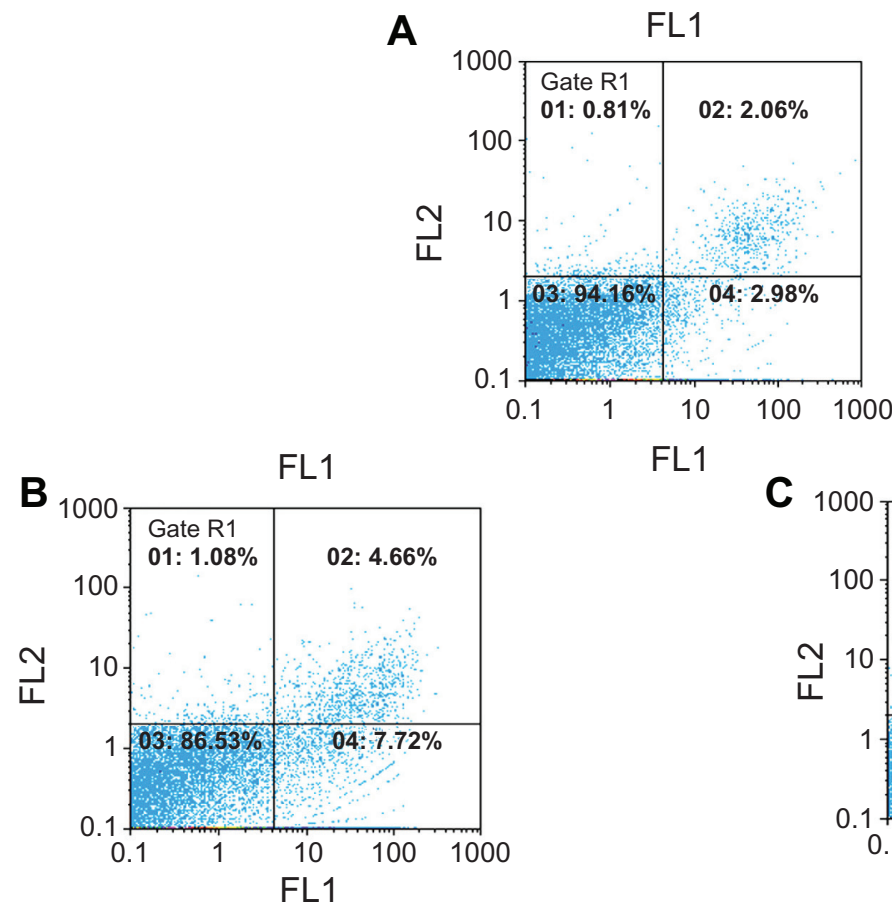

FL1
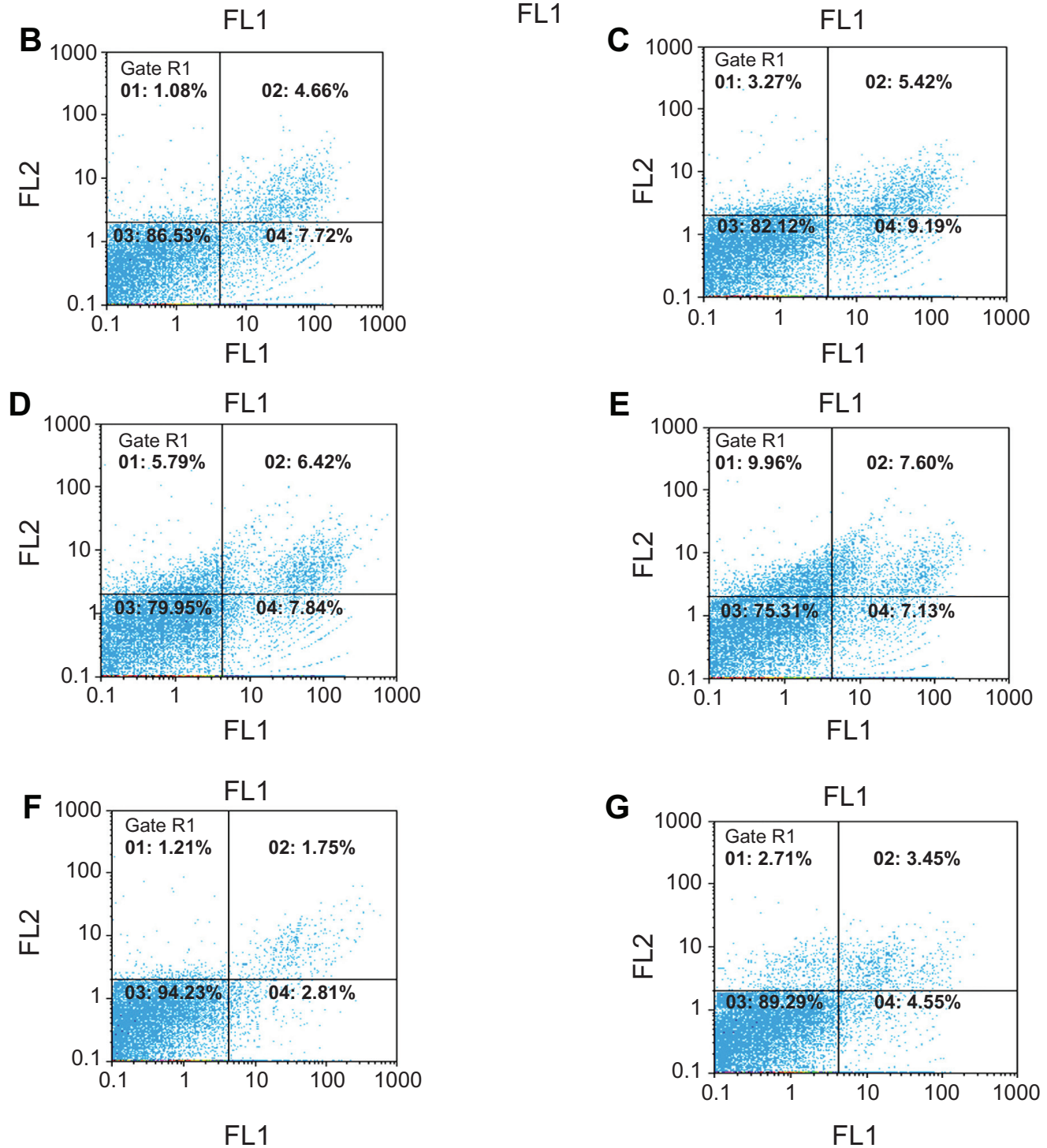

FL1

Figure 8 Dot plot diagrams related to the amount of apoptosis-necrosis assays. Impact of the conjugates and free cisplatin (CDDP) at two concentrations on the percentage of apoptosis and necrosis. The data were analyzed with a version 2.4 of Flomax software. (A) negative control, (B) $\mathrm{Gl}(0.75 \mu \mathrm{g} / \mathrm{ml}),(\mathbf{C}) \mathrm{GI}(\mathrm{I} .5 \mu \mathrm{g} / \mathrm{ml}),(\mathbf{D}) \mathrm{G} 2$ (0.75 $\mu \mathrm{g} / \mathrm{ml})$, (E) G2 (I.5 $\mu \mathrm{g} / \mathrm{ml})$. (F) Platinum $(0.75 \mu \mathrm{g} / \mathrm{ml})$, (G) Platinum ( $1.5 \mu \mathrm{g} / \mathrm{ml})$. Lower right: apoptotic cells, Upper right: necrotic and secondary apoptotic cells, Lower left: viable cells, Upper left: damaged cells.

the $\mathrm{G} 2+\mathrm{Pt}$ conjugate may allow easier recognition and entry to the cell without requiring extra energy. In addition, this greater negative charge of the $\mathrm{G} 2+\mathrm{Pt}$ provides an increased number of sites being able to interact with the positively charged components of the cell membrane in comparison with the G1+Pt.
This causes the $\mathrm{G} 2+\mathrm{Pt}$ conjugate to penetrate the cell more easily at an increased rate this could be another factor contributing to the increased cytotoxicity of the G2+Pt conjugate.

The hemolysis assay was used as a test for the interaction of compounds with the erythrocytes. Regarding the 
results, exactly the same hemolysis behavior was observed for all the conjugates except for the G2+Pt complex at the concentration of $6 \mu \mathrm{g} / \mathrm{mL}$. This concentration showed an increment in the hemolysis only $\sim 1.5 \times$ against the negative control. Although relatively good hemocompatibility for the conjugates, complementary hemocompatibility was observed tests should be performed for better understanding of the hemotoxicity behaviors of these conjugates. With hemolysis results such as these there is little chance of clot formation which might continue in the blood circulation when using these conjugates in vivo eg, in the parenteral form.

Based on the results of the apoptosis-necrosis assays, both of the mechanisms are attributed to cell death, and a concentration dependency was observed for cisplatin and its derivatives. Also, greater toxicity (the percentage of apoptosis and necrosis $\sim 1.5 \times$ ) was observed for the conjugates as compared to the parent cisplatin. Overall, the mode of cell death is very important in cancer therapy as most of the resistances which are observed in cancer cell lines are due to apoptotic-related cell death. ${ }^{28}$ The immunostimulatory impact of the products originating from necrotic cells may stimulate a macrophage-mediated antitumor response (contrary to apoptosis) and may produce an effective immune response by dendritic cells which must also be considered in the final therapeutic outcomes of antitumor drugs. ${ }^{29,30}$ Therefore, it seems possible that necrotic cell death could be more effective than apoptosis in cancer therapy. Certainly, it would be reasonable to consider the bi-lateral effects of necrosis in being an effective cancer treatment and the more accomapnied unwanted inflammatory reactions which may aggravate clinical tolerances to these drugs in patients during the treatment period. Thus, a balance must be made between the two processes of cell death. With respect to the greater potencies according to the more apoptosis-necrosis observed for the G2+Pt and the G1+Pt complexes compared to those of cisplatin, these compounds seem to be able to meet the necessary prerequisites for being good antitumor candidates against the cancer cell lines tested.

\section{Conclusion}

Regarding the conjugates of cisplatin tested during this study and the in vitro results gained for their cytotoxicity, hemototoxicity, and nontoxicity of the dendrimers, it is hoped that these conjugates would be able to maintain the observed potency in vivo and retain the parent drug conjugated at the surface of the dendrimers in the physiologic plasma condition. This property can prohibit the unwanted release of free platinum before reaching the target site ie, tumor cells; to prevent the undesirable systemic toxicity, preclude excessive plasma protein binding with cisplatin, and ultimately exhibit a more repeatable, reproducible, and successful treatment in comparison with treating a patient with free cisplatin. Such drawbacks have been considered to be the main causes of the resistance mechanisms of cisplatin. ${ }^{10}$ Furthermore, the conjugates, especially the G2+Pt conjugate, exhibited more cytotoxic potential in comparison with the parent drug in most of sensitive and resistant cell lines. Therefore, less of the conjugated parent drug would be required to obtain a similar therapeutic effect when compared with cisplatin treatment alone. It would therefore follow that such treatment could be attained with reduced systemic toxicity which is usually seen with this traditional antitumor agent. In brief, future in vivo studies will be able to clarify the potentiality of these entities in the cure of both sensitive and resistant cancerous cells.

\section{Acknowledgements}

Special thanks are given to Tehran University of Medical Sciences for the financial supports of this study.

\section{Disclosures}

The authors have no conflicts of interest that are directly relevant to the content of this review.

\section{References}

1. Ringsdrorf H. Structure and properties of pharmacologically active polymers. J Polym Sci Polymer Symp. 1975;51:135-153.

2. Caiolfa VR, Zamai M, Fiorini A, et al. Polymer-bound camptothecin: initial biodistribution and antitumor activity studies. J Control Release. 2000;65:105-119.

3. Malik N, Evagorou EG, Duncan R. Dendrimer-Platinate: a novel approach to cancer chemotherapy. Anti-cancer Drugs. 1999;10(8):767-776.

4. Roy R. Recent developments in the rational design of multivalent glycoconjugates. Top Curr Chem. 1997;187:241-274.

5. Duncan R, Izzo L. Dendrimer biocompatibility and toxicity. $A d v$ Drug Del Rev. 2005;57:2215-2237.

6. Howell BA, Fan D, Rakesh L. Nanoscale dendrimer-platinum conjugates as multivalent antitumor drugs. In: Abd-EL-Aziz AS, Carraher CEJ, Pittman CUJ, Zeldin M, editors. Inorganic and organometallic macromolecules: design and applications. 1st ed. New York: Springer; 2007:269-294.

7. Zutphen SV, Reedijk J. Targeting platinum antitumor drugs: overview of strategies employed to reduce systemic toxicity. Coord Chem Rev. 2005;249(24):2845-2853.

8. Rosenberg B, Vancamp L, Trosko JF, Mansour VH. Platinum compound: a new class of potent antitumor agents. Nature. 1967;222:385-396.

9. Kartalou M, Essiqmann JM. Mechanisms of resistance to cisplatin. Mutat Res. 2001;478(1-2):23-43.

10. Stewart DJ. Mechanism of resistance to cisplatin and carboplatin. Crit Rev Oncol Hematol. 2007;63(1):12-31.

11. Horacek P, Drobnik J. Interaction of cis-diamminedichloroplatinum (II) with DNA. Biochim Biophys Acta. 1971;254:341-347. 
12. Micetich KC, Barnes D, Erickson LC. A comparative study of the cytotoxicity and DNA damaging effects of cis-(diammino)(1,1cyclo-butanedicarboxylato-platinum (II) on L1210 cells. Cancer Res. 1985;45:4043-4047.

13. Hanigan MH, Devarajan P. Cispaltin nephrotoxicity: molecular mechanisms. Cancer Ther. 2003;1:47-61.

14. Groth S, Nielson H, Sorenson JB, et al. Acute and long term nephrotoxicity of cisplatin in man. Cancer Chemother Pharmacol. 1986;17: 191-196.

15. Namazi H, Adeli M. Novel linear-globular thermoreversible hydrogel ABA type copolymers from dendritic citric acid as the A blocks and poly (ethyleneglycol) as the B block. Eur Polym J. 2003;39:1491-1500.

16. Namazi H, Adeli M. Dendrimers of citric acid and poly (ethylene glycol) as the new drug-delivery agents. Biomaterials. 2005;26:1175-1183.

17. Blagosklonny MV. Prospective strategies to enforce selectively cell death in cancer cells. Oncogene. 2004;23:2967-2975.

18. Mosmann T. Rapid colorimetric assay for cellular growth and survival: application for proliferation and cytotoxicity assay. J Immuno Methods. 1983;65:55-63.

19. Adeli M, Mirab N, Shafiee Alavidjeh M, Sobhani Z, Atyabi F. Carbon nanotubes-graft-polyglycerol: Biocompatible hybrid material in nanomedicine. Polymer. 2009;50:3528-3536.

20. Iwasaki Y, Ijuin M, Mikami A, Nakabayashi N, Ishihara K. Behavoir of blood cells in contact with water-soluble phospholipid polymer. J Biomed Mater Res. 1999;46:360-367.

21. Vermes I, Haanen C, Steffen S, Nakken H, Reuteligsperger C. A novel assay for apoptosis: flow cytometry detection of phosphatidyl serine expression on early apoptotic cells using fluorecein-labeld Annexin J Immuno Methods. 1995;184:39-51.
22. Gianasi E, Buckley RG, Latigo J, Wasil M, Duncan R. HPMA copolymers platinates containing dicarboxylato ligands, preparation, characterisation and in vitro and in vivo evaluation. J Drug Target. 2002;10(7):549-556.

23. Amaravadi RK, Thompson CB. The roles of therapy-induced autophagy and necrosis in cancer treatment. Clin Cancer Res. 2007;13(24): 7271-7279.

24. Ferruti P, Ranucci E, Trotta F, Gianasi E, et al. Synthesis, characterisation and antitumor activity of platinum(II) complexes of novel functionalized poly (amidoamine)s. Macromol Chem Phys. 1999;200: 1644-1654.

25. Ren Y, Zhang H, Huang J. Synthesis and cytotoxic activity of platinum complex immobilized by branched polyethylene glycol. Bioorg and Med Chem Lett. 2005;1:4479-4483.

26. Mistry P, Kelland LR, Loh SY, et al. Comparison of celluar accumulation and cytotoxicity of cisplatin with that of tetra platinum and amminiedibutyratodichloro(cyclohexylamine)platinum (IV) (JM221) in human ovarian carcinoma cell lines. Cancer Res. 1992;52:6188-6197.

27. Berg JM, Tymoczko JL, Stryer L. Biochemistry. 5th ed. New York: WH Freeman and Company; 2002. p. 465-484, 494-501.

28. Ng CP, Bonavida B. A new challenge for successful immunotherapy by tumors that are resistant to apoptosis: two complementary signals to overcome cross resistance. Adv Cancer Res. 2002;85:145-174.

29. Galluci S, Lolkema M, Matzinger P. Natural adjuvants: endogenous activators of dendritic cells. Nat Med.1999;5:1249-1255.

30. Sauter B, Albert ML, Francisco L, et al. Consequences of cell death: exposure to necrotic tumor cells, but not primary tissue cells or apoptotic cells, induces the maturation of immunostimulatory dendritic cells J Exp Med. 2000;191:423-430.
International Journal of Nanomedicine

\section{Publish your work in this journal}

The International Journal of Nanomedicine is an international, peerreviewed journal focusing on the application of nanotechnology in diagnostics, therapeutics, and drug delivery systems throughou the biomedical field. This journal is indexed on PubMed Central, MedLine, CAS, SciSearch $₫$, Current Contents $₫ /$ Clinical Medicine,

\section{Dovepress}

Journal Citation Reports/Science Edition, EMBase, Scopus and the Elsevier Bibliographic databases. The manuscript management system is completely online and includes a very quick and fair peer-review system, which is all easy to use. Visit http://www.dovepress.com/ testimonials.php to read real quotes from published authors 